\title{
Strength and electronic structure
}

\author{
K GOVINDA RAJAN
}

Materials Science Laboratory, Reactor Research Centre, Kalpakkam 603 102, India

\begin{abstract}
The main attribute of a solid is its resistance to deformation, or its 'strength'. We discuss first the interpretation of the strength parameter. The current situation with regard to the central problem of providing a microscopic description of the strength parameter is briefly reviewed. Electrons in metais provide the cohesion, so that an understanding of the role played by electronic structure in the strength attribute should lead to practical hints for building stronger materials. The useful 'aircraft alloy' $(\mathrm{Ti}+\mathrm{AI}+\mathrm{V})$ illustrates one such important relationship, viz., that the addition of a non $d$-character metal to a $d$-electron host strengthens the latter. Again, metals are distinguished from non-metals by the Fermi surface they possess, and it is interesting to examine any possible relationship between the anisotropy of the Fermi surface with the observed anisotropy in hardness (or yield strength). Next, we turn to cleavage, and point out that the assumption that it is the exact opposite of cohesion faces objections. Cohesion is an average property, whereas cleavage is a crack-tip phenomenon. Finally, among the processes familiar to the metallurgist wherein a metal is hardened, electron-moderated mechanisms have been identified in at least two cases, and we conciude with a brief account of these.
\end{abstract}

Keywords. Strength; cohesive energy; electronic energy; electron-mediated interactions; strengthening mechanisms.

“... merely corroborative details to lend an aspect of verisimilitude to what would otherwise be a bald and unconvincing narrative ..."

Gilbert and Sullivan-The Mikado

\section{Introduction}

A solid is characterized by its resistance to deformation, or its strength. In particular, the ability of a solid to resist shearing forces is used to distinguish it from a fluid. Three broad characteristics can be recognized when the meaning of 'strength' is considered. Resilience or elasticity, when subjected to transient forces-that is, how strongly does the solid attempt to regain its original shape if a force is applied to it for a time and then removed? The Young's modulus, which is a measure of such resistance, varies over three orders of magnitude $\left(10^{10}-10^{13} \mathrm{dyn} / \mathrm{cm}^{2}\right)$. Resistance to flow is the next characteristic and it concerns the question: how much applied force is requirea to obtain a particular rate of flow? Flow resistance varies even more-from $10^{5}$ to $10^{12} \mathrm{dyn} / \mathrm{cm}^{2}$-and the measured rates of flow vary over 12 orders of magnitude. Third, by applying sufficient tension, solids can be pulled apart into two pieces. How much applied force is required to fracture a solid? Fracture resistance is the resistance of a material to crack propagation. It is measured in terms of the fracture surface energy, which may be as little as $10 \mathrm{dyn} / \mathrm{cm}$ or as high as $10^{8} \mathrm{dyn} / \mathrm{cm}$, i.e., varies over 7 orders of magnitude.

Owing to these diverse and variable aspects, the problem of strength is one of great complexity. Strength depends on the collective response of a crystal to applied stress, so 
that its description is quite involved. That is, strength depends on the behaviour of aggregates of particles, and the central problem in the science of strength has been to learn how to describe mechanical behaviour in terms of the fundamental properties of matter, so that the shapes of stress-strain or strain-time curves and their dependence on loading conditions can be predicted from a small number of experimental relationships (Gilman 1969).

Turning to metals, we know that the properties of metals arise from the behaviour of the free electrons, and their interaction with the metallic ions. Much attention has therefore been directed to the properties of electrons (in particular, the free electrons) in metals, and features such as electrical conduction, magnetic properties and structure in metals have been understood. This success provides the motivation to look into the question of whether progress with regard to the strength attribute too can be made using this approach.

\section{Perfect cleavage}

We begin by discussing how the strength of a solid is calculated in order to understand the role of electronic structure.

The separation of a crystal into two or more parts by the propagation of a geometrically plane crack through the solid is called cleavage. Perfect cleavage is portrayed in figure 1. A crystal is stressed in tension vertically to the point of theoretical cohesive strength (figure 1a), and then this stress is exceeded simultaneously at every one of the atomic bonds across the cleavage plane extending interminably and mathematically all the way across the stressed crystal (figure 1b), with no intermediate stages. The crystal goes directly from cohesion to cleavage with no crack propagation direction. The more recent, and present, concept of perfect cleavage is that the elastic bonds connecting the atoms are individually overcome one by one in chronological order, as shown in figure 1c. In this visualization, perfect cleavage is the propagation of an atomically sharp crack with the applied stress overcoming the elastic stresses in a purely elastic manner, and with the atoms separating in geometrically opposite directions, along the direction of the tensile stress.

\subsection{Ideal strength of a crystalline solid}

The maximum stress required to produce, in an ideal crystal, a perfect cleavage of the form imagined above is called the ideal strength or the theoretical strength of a solid

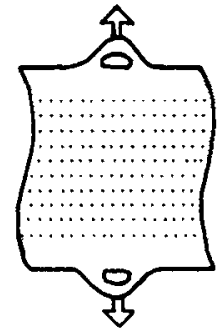

(a)

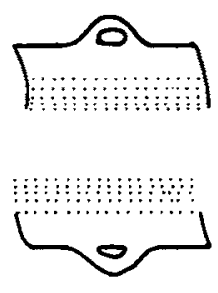

(b)

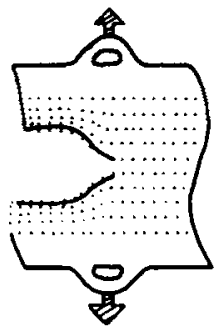

(c)

Figure 1. Perfect cleavage: (a) and (b) Original picture; (c) present concept of perfect cleavage. 
(Ts). Before the development of the dislocation theory of solids, this problem was of considerable interest because calculated values of the strength were typically greater than experimental values by a factor of 100 or more. Even at the present time, this problem is of theoretical and practical importance, as a knowledge of the Ts is useful in determining the stress distribution and other properties in the region at the tip of a crack; in determining the dislocation core radii; in defining the point at which coherency breakdown occurs at a particle-matrix interface; and in providing insight into the phenomenon of melting. The reviews by Milstein (1982) and Macmillan (1972) exemplify these points. Macmillan also reviews the practical approach to the measurement of Ts. The measurements are carried out either on specially grown whiskers or on single crystals of materials in which the dislocation density is very small $\left(\sim 10^{1}-10^{2} \mathrm{~cm}^{2}\right)$. Table 1 provides a comparison between the calculated and measured strength for a few solids.

A simple way of estimating the cleavage stress of a perfect crystal is as follows (Kelly 1973). It is assumed that the crystal is pulled at $T=0$, and the restoring force per unit area between two adjacent atomic planes is assumed to vary with distance according to $\sigma=K \sin \pi / a\left(x-a_{0}\right)$, where $a_{0}$ is the equilibrium separation between the atomic planes, and $a$ is the range of the interatomic force. The latter is evaluated by setting

$$
2 \gamma=\int_{a_{0}}^{a_{0}+a} \sigma \mathrm{d} x
$$

at cleavage, $\gamma$ being the surface energy of the solid; the factor 2 appears because a pair of surfaces is created when cleavage occurs. Thus $a=\pi \gamma / K$. The maximum value of $\sigma$ is identified as the rs of the solid, so that we have simply $\sigma_{\max }=K . K$ is evaluated from the definition of the Young's modulus $\Upsilon$ for the appropriate direction in the solid, namely, $\Upsilon=a_{0} \mathrm{~d} \sigma / \mathrm{d} x$. For $x \simeq a$, this gives

$$
\Upsilon=K \pi \frac{a_{0}}{a} \cos \frac{\pi}{a}\left(a-a_{0}\right) \simeq \frac{K \pi a_{0}}{a}
$$

\begin{tabular}{|c|c|c|c|c|c|c|}
\hline \multicolumn{4}{|c|}{ Calculated values of $\mathrm{Ts}$} & \multicolumn{3}{|c|}{ Experimental data } \\
\hline \multirow{2}{*}{ Material } & \multirow{2}{*}{$\begin{array}{l}\text { Tensile } \\
\text { direction }\end{array}$} & \multicolumn{2}{|c|}{$\begin{array}{l}\text { Theoretical strength } \\
\text { (calculated) } \times 10^{-11} \mathrm{dyn} / \mathrm{cm}^{2}\end{array}$} & \multirow{2}{*}{ Material } & \multirow{2}{*}{$\begin{array}{c}\text { Max. tensile stress } \\
\sigma_{\max } \times 10^{-10} \mathrm{dyn} / \mathrm{cm}^{2}\end{array}$} & \multirow{2}{*}{$\frac{\sigma_{\max }}{\widehat{\Upsilon}} \approx$} \\
\hline & & equation $(1)^{*}$ & equation (2) & & & \\
\hline $\mathbf{A g}$ & $\langle 111\rangle$ & $2 \cdot 4(0.20)$ & - & Whiskers & & \\
\hline $\mathrm{Ag}$ & $\langle 111\rangle$ & $2.7(0.25)$ & $\ldots$ & $\mathrm{Ag}$ & $1.73\langle 100\rangle$ tension & $0-040$ \\
\hline $\mathrm{Cu}$ & $\langle 111\rangle$ & $3.9(0.20)$ & 3.796 & $\mathrm{Cu}$ & $2.94\langle 111\rangle$ tension & 0.028 \\
\hline $\mathrm{Cu}$ & $\langle 100\rangle$ & $2.5(0.51)$ & $3 \cdot 60$ & $\mathrm{Fe}$ & $13 \cdot 10\langle 111\rangle$ tension & $0-049$ \\
\hline $\mathbf{w}$ & $\langle 100\rangle$ & $6.1(0.22)$ & & $\mathrm{Cd}$ & $2.80\langle 1120\rangle$ tension & 0.040 \\
\hline$\alpha-\mathrm{Fe}$ & $\langle 100\rangle$ & $3.0(0 \cdot 34)$ & & & & \\
\hline$\alpha-\mathbf{F e}$ & $\langle 111\rangle$ & 4.6 & & Crystal & & \\
\hline $\mathrm{C}($ dia $)$ & $\langle 111\rangle$ & 20.5 & & $\mathrm{Ge}$ & 7.12 & 0.05 \\
\hline $\mathrm{Si}$ & $\langle 111\rangle$ & $3 \cdot 2$ & & & & 0.08 \\
\hline $\mathrm{Ni}$ & $\langle 100\rangle$ & & $2 \cdot 3$ & & & \\
\hline
\end{tabular}

Table 1. Comparison of calculated and measured values of the strength of a few solids.

* Values indicated in parentheses are the calculated strains. 
so that we have, finally,

$$
\sigma_{\max }=\left(\Upsilon \gamma / a_{0}\right)^{1 / 2}
$$

This simple treatment indicates that the strength goes as $\sqrt{\Upsilon}$ and $\sqrt{\gamma}$. The values of strengths obtained from this approach are indicated in table 1.

\subsection{More exact calculations of $T S$}

After Born's work on the necessary conditions for the thermodynamic stability of a crystal lattice with respect to arbitrary (but small) homogeneous deformations, many attempts have been made to refine the calculations of the theoretical strength (Esposito et al 1980; Milstein 1982). The current approach is to proceed along the following lines: (a) The solid under consideration is defined by specifying its crystal structure, parameters of the unit cell, etc. (b) The interaction between the ions and electrons is specified next. For simplicity, pair potentials (Lennard-Jones, Morse, etc.) are used. However, for metals where there is a clear deviation from the Cauchy relation $C_{12}=C_{44}$, the pair potential approximation is not applicable. Recently, the so-called "ab-initio potential" method for the calculation of the ts has been developed to overcome the limitations of the pair-potential approximation (Carlsson et al 1980). (c) The Born stability criteria are applied, and the suitability of the potential is checked. (d) The cohesive energy per atom (or unit cell) is calculated as a function of the parameters of the potential and other geometric parameters. (e) The unit cell is stretched, and the energy is calculated as a function of the strain. (f) Considering for the moment a cubic crystal with lattice parameter $a_{0}$ (thus $a_{1}=a_{2}=a_{2}=a_{0}$ ), it is clear that a stretch, for example along [100], will impart a tetragonal distortion. For computational ease the deformation is imagined to increase $a_{1}$ and decrease $a_{2}$ and $a_{3}$ in such a manner as to keep $a_{2}=a_{3}$ and the transverse stress $\sigma_{2}=\sigma_{3}=0$. In practice, this is achieved by an iterative technique. The relevant stress $\sigma_{1}$ is calculated from the expression

$$
\sigma_{1}=\frac{4}{a_{0}^{3}} \frac{\partial U}{\partial\left(a_{1} / a_{0}\right)}
$$

where $U$ is the cohesive energy. The theoretical strength is identified as

$$
\mathrm{TS}=\left(\sigma_{1}\right)_{\max } .
$$

Figure 2 gives an idea of the way $\sigma_{1}$ varies with extension along [100] in nickel. The maximum $\sigma_{1}$ value is the TS, and the corresponding $a_{1}$ value gives the strain at fracture. Obviously, the cleavage process is imagined to be the exact opposite of cohesion, with all bonds snapping simultaneously when $\sigma_{1}$ reaches $\left(\sigma_{1}\right)_{\max }$.

Further details may be found in the references listed at the end (e.g., Milstein 1982). Table 1 also contains the rs values calculated by these more exact methods. It is interesting to note that even the naive calculations outlined in $\$ 2.1$ are already in reasonably good agreement with the observed values. The more exact calculations, in addition to improving this agreement, also help us in understanding the correlation between the electronic structure and strength of metals. 


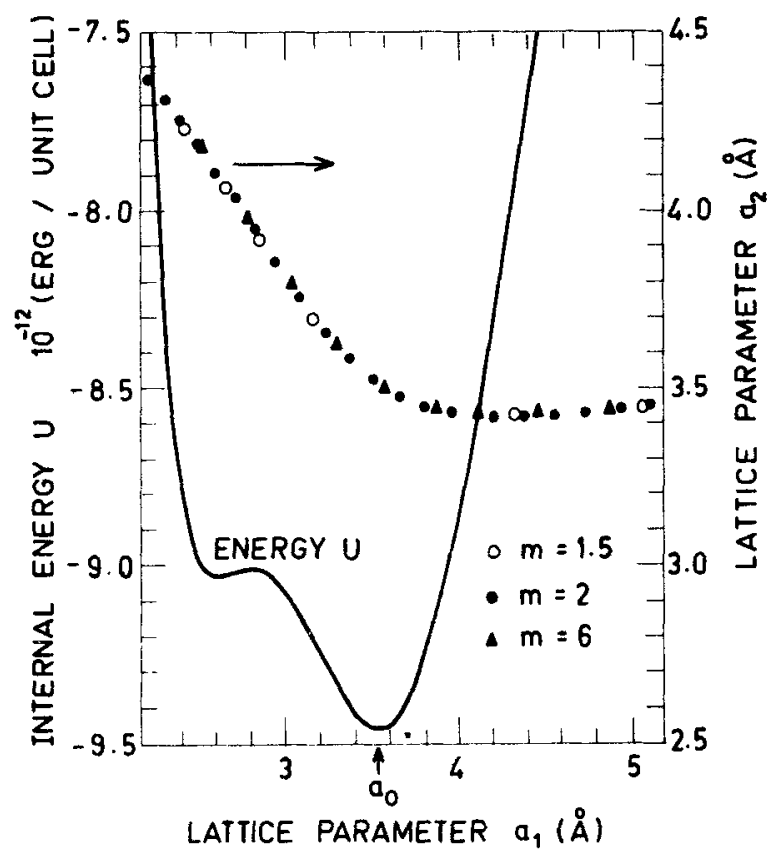

(a)

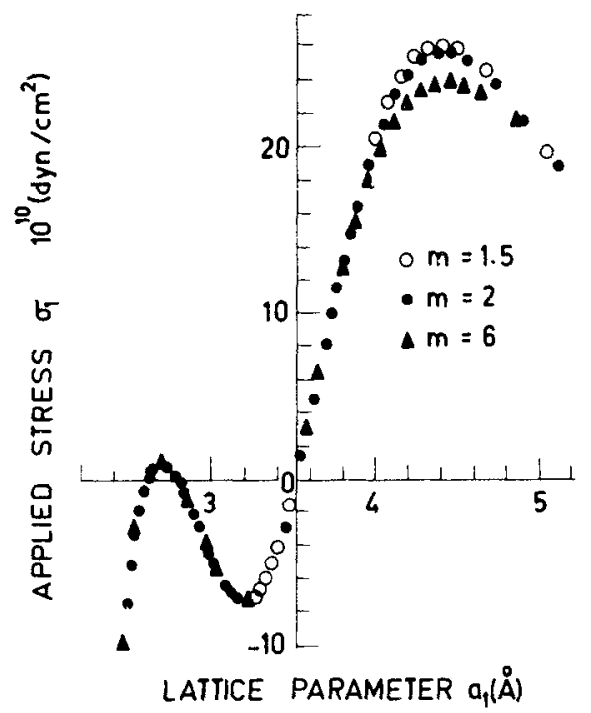

(b)

Figure 2. (a) The internal energy (cohesive energy) as a function of the axial lattice parameter $a_{1}$ for [100] uniaxial loading in fcc Ni. (b) Computation of the true axial stress $\sigma_{1}$ as a function of $a_{1}$ for [100] loading. The maximum value of $\sigma_{1}$ is the theoretical strength. The calculations were performed using a Morse potential $\phi(r)=D\left[\exp \left\{-2 m \alpha\left(r-r_{0}\right)\right\}-2 \exp \right.$ $\left.\left\{-m \alpha\left(r-r_{0}\right)\right\}\right]$. Results for a few values of $m$ are shown (after Milstein 1982). 


\section{Cohesion and electronic structure}

We have seen that in strength calculations, the cohesive energy of the solid has to be first determined. It therefore becomes necessary to see how the electronic structure enters the calculation of cohesive energy, for a better understanding of the relationship between strength and electronic structure (especially in metals).

The calculations of the total energy of a metallic structure is formulated as the determination of the energy of an assembly of $N$ ions (each with volume $\Omega$ ) at sites $\mathbf{r}_{n}$ arranged on a crystal lattice, and $N Z$ valence electrons. The total energy $U$ is first separated into the energy of the ions, which is purely potential and comes from their mutual repulsion, and that of the electrons, which contains both kinetic and potential terms. The energy of an electron with momentum $k$ is given (to a second order of approximation) by

$$
E(k)=\frac{\hbar^{2} k^{2}}{2 m}+V_{0}+\sum_{q}^{\prime} \frac{V_{q}^{2}}{E_{k}-E_{k+q}},
$$

in standard notation. To obtain the total energy of the electrons, we must sum $E(k)$ over all occupied states, i.e., over all $k \leqslant k_{F}$, the Fermi momentum of the electrons. The first two terms on the right in (3) are free-electron terms, this being the zeroth approximation for the perturbation theory. All such terms are structure-independent. The first term is the kinetic energy of the free electron gas, and the second is the average value of the lattice potential. Both terms depend upon the total volume. All the structure dependence of $E(k)$ resides in the third term, which contains the strengths of atomic potentials' expressed in some suitable form. If the potentials are weak, a secondorder correction is adequate and this leaves $E(k)$ only slightly structure-dependent. The corresponding mechanical characteristic is softness; e.g.: Na, $\mathrm{K}$, etc. As the strength of the atomic potentials in a sequence of metals increases more terms have to be added to $E(k)$, making it more and more structure-dependent. As a result, these metals fall into a sequence of increasing hardness (or strength). It has been suggested that the parameter $E_{g} / \Delta$ can also be used as a measure of atomic potential strength, where $E_{g}$ is the width of a low-lying band gap at the zone boundary and $\Delta$ is the width of the energy band itself.

The cohesive energy of a metal can be written as a sum

$$
U=U_{0}+U_{s}
$$

of a structure-independent part $U_{0}$, and a structure-dependent term $U_{s}$. The former is given by

$$
\begin{aligned}
U_{0} & =U_{e}+Z V_{0}+W_{E} \\
& =\frac{3}{5} Z E_{F}+Z V_{0}-0.9 \frac{Z^{2} e^{2}}{R} .
\end{aligned}
$$

The first two terms represent the free electron energy averaged over all the occupied states. ( $E_{F}$ is the free electron Fermi energy.) $Z V_{0}$ is the mean value of the pseudopotential in a sphere of $\Omega$, i.e. the mean potential energy per electron multiplied by the number of electron per ion. $W_{E}$ is the electrostatic energy of the ions in the uniform free electron gas. In determining its value, the ions are imagined to be spheres immersed in the electron gas. When crystal structures are taken into account, the geometric factor 0.9 is modified to other values very close to 0.9 . Correction terms like 
Table 2. Comparison between the observed values of the cohesive energy $U$ with the calculated values of the structure independent part of $U$.

\begin{tabular}{lcl}
\hline Element & $\begin{array}{c}\text { Measured } \\
\text { calculated } \\
\text { cohesive } \\
\text { energy (ry) }\end{array}$ \\
\hline $\mathrm{Li}$ & 0.513 & 0.512 \\
$\mathrm{Na}$ & 0.457 & 0.460 \\
$\mathrm{~K}$ & 0.369 & 0.388 \\
$\mathrm{Be}$ & 0.995 & 1.13 \\
$\mathrm{Mg}$ & 0.852 & 0.892 \\
$\mathrm{Zn}$ & 0.991 & 1.05 \\
$\mathrm{Cd}$ & 0.937 & 0.993 \\
$\mathrm{Al}$ & 1.32 & 1.38 \\
$\mathrm{Sn}$ & 1.69 & 1.77 \\
$\mathrm{~Pb}$ & 1.73 & 1.81 \\
\hline
\end{tabular}

the electrostatic self-energy, that is, the interaction of the electron gas with itself, also have to be considered. The structure-independent energy $U_{0}$ is thus

$$
U_{0}=\frac{3}{5} Z E_{F}+Z V_{0}-0.9 \frac{Z^{2} e^{2}}{R}-\frac{e^{2}}{2 Z} \int \frac{\rho(\mathbf{r})(r)}{\left|\mathbf{r}-\mathbf{r}^{\prime}\right|} \mathrm{d} \mathbf{r} \mathrm{d} \mathbf{r}^{\prime} .
$$

Further correction terms to allow for correlation and exchange effects among the conduction electrons and for the exclusion of conduction electrons from the core, enable $U_{0}$ to be determined more accurately.

A comparison (table 2) between the observed and calculated cohesive energies for a number of metals shows that the structure-independent energy dominates the volume dependence of the total energy; so that, by choosing a particular pseudopotential form, $U_{0}$ can be evaluated as a function of $\Omega$. From this, the structure, compressibility and strength follow readily.

The structure-dependent energy $U_{s}$ has the following terms:

$$
U_{S}=U_{\mathrm{BS}}+\Delta W_{E}
$$

The band structure energy $U_{\mathrm{BS}}$ is the change in energy in going from the free electron model to one containing a periodic potential. $\Delta W_{E}$ is a structure-dependent correction to $W_{E}$. In one dimension, if the Fermi level lies just below a band gap, the effect of the band structure is a lowering of the energy below the free electron level. If the Fermi level were far above a band gap, the net effect would leave the free electron energy unaltered. However, the actual Fermi surfaces in metals and other crystals always lie close to the first few reciprocal lattice vectors so that the effect of the band gaps always decreases the total energy, i.e. $U_{\mathrm{BS}}$ is always negative. Figure 3 shows this schematically. This structure-dependent part of the electron energy can be expressed as

$$
U_{\mathrm{BS}}=\sum_{k<k_{F}} \sum_{g}^{\prime} \frac{V_{g} V_{g}^{*}}{E_{k}-E_{k+g}} .
$$

This is a perturbation-theoretic result, and is not accurate close to the Brillouin zone boundary-it diverges when the Bragg condition is exactly satisfied. The way the 
structure controls $U_{\mathrm{BS}}$ can be seen by writing the final form of $U_{\mathrm{BS}}$ as

$$
U_{\mathrm{BS}}=\sum_{\substack{g \\ g \neq 0}}^{\prime}[S(g)]^{2}[V(q)]^{2} \chi(q) \varepsilon(q),
$$

where $S(g)$ is zero except at reciprocal lattice vector points and is thus the sole repository for the influence of the structure. The remaining factors are characteristic only of the nature of the atoms in question, and they are usually collectively termed the energywavenumber characteristic $F(q)$ :

$$
F(q)=V(q)^{2} \chi(q) \varepsilon(q)
$$

$F(q)$ has the form as shown in figure 4 for various metals.

The theory described so far has been used by metal physicists to understand the crystal structure of metals, to provide the basis for the Hume-Rothery rules, and to explain the stability of alloy phases at the absolute zero of temperature (Harrison 1966; Wilkes 1973). Calculation of the theoretical strength has been attempted only for copper and fcc iron. Once $U$ is calculated the calculation of the strength proceeds on the lines explained in $\S 2.2$. Table 3 indicates the values of theoretical strengths obtained for copper using three different potentials. Obviously, this approach of treating the

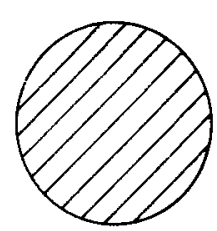

(a)

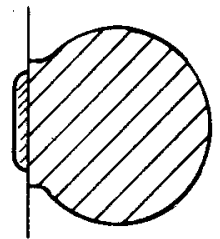

(b)

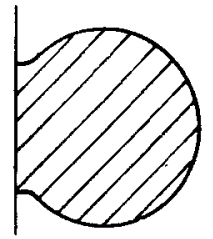

(c)

Figure 3. (a) Free electron Fermi surface-a sphere. (b) The Fermi sphere intersected by a Brillouin zone. This gives rise to little energy change. (c) Sphere touching plane-energy gain.

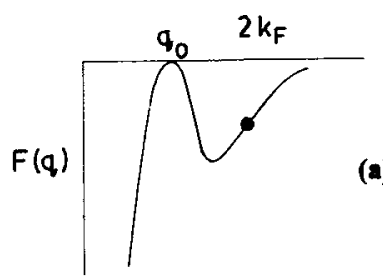

(a)

Figure 4. (a) Schematic diagram of energy-wavenumber characteristic. (b) Energy-wavenumber characteristic for some metals.

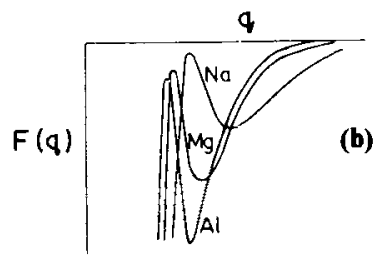


Table 3. The theoretical strength of copper calculated using three different model potentials.

\begin{tabular}{|c|c|c|}
\hline Potential & $\begin{array}{c}\text { Theoretical } \\
\text { strength, } \sigma_{t}\left(\text { dyn } / \mathrm{cm}^{2}\right)\end{array}$ & $\begin{array}{c}\text { Strain } \\
\varepsilon_{t}=\sigma_{t} / \Upsilon\end{array}$ \\
\hline Nonself-consistent KKR & $5.5 \times 10^{11}(\Upsilon / 2.3)$ & 0.5 \\
\hline \multicolumn{3}{|l|}{ Self-consistent ASW with } \\
\hline Ewald correction & $(Y / 40)$ & $0 \cdot 3$ \\
\hline Pair potential & $(X / 4 \cdot 0)$ & 0.5 \\
\hline
\end{tabular}

electrons as nearly free cannot be used when dealing with transition and rare-earth metals, as these possess narrow $d$ and $f$ bands respectively.

It may be observed from tables 1 and 3 that while there is satisfactory agreement between the computed and observed $\sigma_{t}$ values, the calculated values of the strains $\varepsilon_{t}$ at failure are nearly an order of magnitude greater than those found experimentally. Brenner's (1956) early experiments on single crystal copper whiskers indicated for [111] tension, a maximum stress $\sim \Upsilon / 40$, where $\Upsilon$ is the measured Young's modulus for polycrystalline copper, and a strain of only $0.03(3 \%)$. Brenner noticed that in his experiments, all the specimens did not cleave, but sheared apart. It might also be of interest to note that in another study (McQueen and Marsh 1962), flat single crystal samples of $\mathrm{Cu}$ were subjected to shock waves of sufficient intensity to cause fracture. Because of the geometry of the experiment, the transverse dimensions of the sample remain essentially fixed. For the [100] orientation, the yield strength obtained was $r / 7.5$. However, the derivation of this value from the known shock-wave intensity involved the use of hydrodynamic equations and equations of state whose validity is difficult to assess.

The substantial difference between the calculated and the observed strains prompts us to examine closely how good the foregoing picture of cleavage is. It is true that in some experiments a shear yield rather than no clean cleavage was observed. Yet, this alone cannot account for the entire difference. It must be that, after all, fracture is not as simple as pure cleavage, which is just the antithesis of cohesion. Finally, it should be mentioned that the strength calculations have so far been performed for a simple cubic unit cell; it is not known at present just how complicated these calculations are for other lower symmetries.

\section{Cohesion vs cleavage}

Cohesion may be described as a process holding the atoms together and cleavage as a process parting the atoms, and it is tempting to imagine these processes being exactly opposite in character. In tensile tests crystals are held together by cohesion until a load is reached where cleavage (or other fracture) occurs. From the measured load and the cross-sectional area one can compute the average force per unit area which existed at the time of fracture. This is the fracture, or cleavage, stress. It is not the cohesive strength; up to the point of fracture, the crystal had cohesion, and every atomic bond across the future cleavage plane was certainly strained in something close to perfectlyelastic tension. The moment a crack formed, however, be it $10 \AA$ or $2000 \AA$, a new condition came into being--the crack tip. In short, cleavage is a crack-tip phenomenon, 
whereas cohesion is an average property of a crystal. It is appropriate to quote Beachem (1978) here: "Cohesive energies, or cohesive forces, do account for the loads to which tensile or bend specimens can be subjected before they break. Once fracture starts, however, events become localized and average properties become less important than local stresses, general microstructures become less important than local crack-tip microstructures, and bulk environments become less important than local crack-tip environments. Those average macroscopic bulk properties which were used in predicting the forces to which the whisker (or other specimen) could be loaded, before fracture, no longer apply. Local properties and events become decisive. Once fracture starts, it is a whole new ball game."

\section{Yield strength, hardness and related parameters}

The hardness of materials is a very useful quantity from the practical viewpoint. It is an empirical parameter; depending on the material, and is measured on a suitable scale. Figure 5 shows how the Vicker's hardness varies across the periodic table for the various elements. The hardness of a crystalline material and its yield strength are proportional to each other (Gilman 1973; Havner 1979). Thus the same trend in variation is expected for the strength attribute. The first prominent peak at around $e / a \sim 7$ lies among the transition metals possessing narrow $d$-bands. The second, higher peak is found among the group IV metalloid elements. This latter peak in the

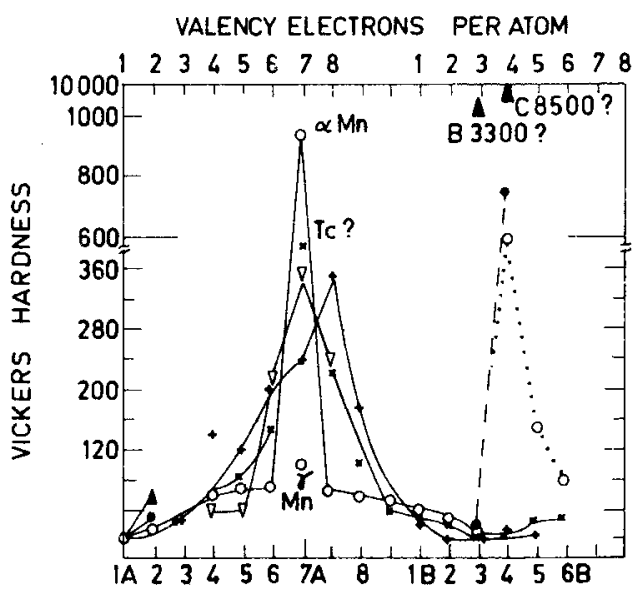

(a)
A Li Be
B C
2s period
- $\mathrm{Na} \mathrm{Mg}$
Al $\mathrm{Si}$
35
- K Ca Sc Ti V Cr Mn Fe Co Ni
Cu $\mathrm{Zn}$ Ga Ge As Se 45
× $\mathrm{Rb} \mathrm{Sr}$ Y $\mathrm{Zr}$ NbMo Tc Ru Rh Pd Ag Cd In Sn Sb Te 5s
+ Cs Ba La Hf Ta W Re Os Ir Pt Au Hg Tl Pb Bi Po 6s "
$\nabla$ Fr Ra Ac Th Pa U Np Pu Am
semi-metals

(b)

Figure 5. Vickers hardness at room temperature for various metals (after O'Neill 1967). 
strength is interpreted in terms of the progressive changeover from metallic to covalent bonding. The second peak will still be there, even if one questions the validity of inclusion of diamond (metastable phase) in the figure.

Extensive data on the hardness of metals and alloys are available in the literature. In recent years, the microhardness parameter of metals and alloys has come to be regarded as the 'strength' microprobe (Westbrook and Conrad 1973). Two recent results concerning the behaviour of microhardness are relevant here and serve to illustrate the subtle interplay between the electronic structure and the strength. The first is the rather surprising correlation found between the microhardness and the semiconductor-tometal transition pressure in group IV elements (Gerk and Tabor 1978). The hardness values of $\mathrm{Ge}, \mathrm{Si}$ and $\mathrm{C}$ (diamond) were found to be 800,1200 and $10^{4} \mathrm{~kg} / \mathrm{mm}^{2}$ respectively. These values are remarkably close to the hydrostatic pressure values necessary to initiate semiconductor-to-metal transition in these elements $(1000,1550$ and $\sim 10^{4} \mathrm{~kg} / \mathrm{mm}^{2}$ ). This indicates that plastic flow has been sustained (ductitity indicates the presence of the metallic phase) in these brittle materials, and is probably because the indentation process involves a large component of hydrostatic stress inhibiting brittle failure. The second point concerns the now-established anisotropy with respect to crystallographic direction of the hardness of crystals. The elegant experiments of Tabnr and his school, especially those of Brookes, have established the scratch hardness technique on an analytical basis, and data on the hardness along different crystallographic directions is available (Brookes 1981; O'Neill 1967; Westbrook and Conrad 1973). Results for copper appear in figure 6. One interprets the anisotropy in terms of the variation in the resolved shear stress for a specified indentor orientation, and therefore with the relevant active slip system. Since hardness and yield strength can be imagined to be directly related in metals, the question arises whether the

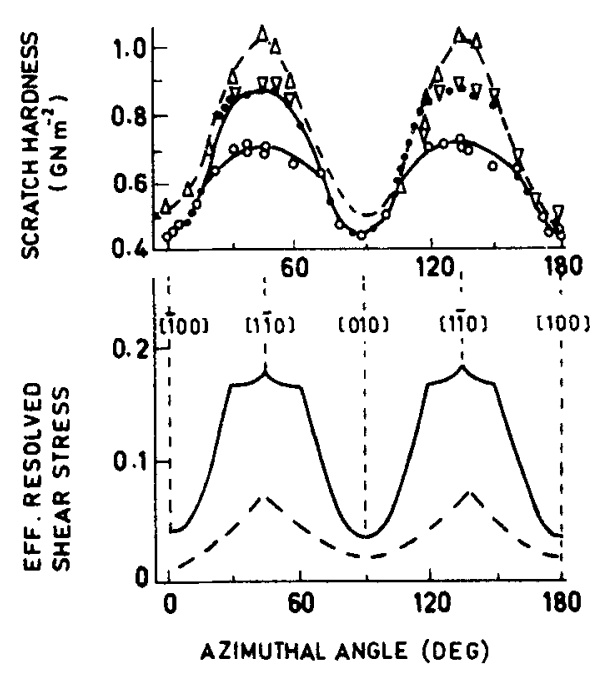

(a)
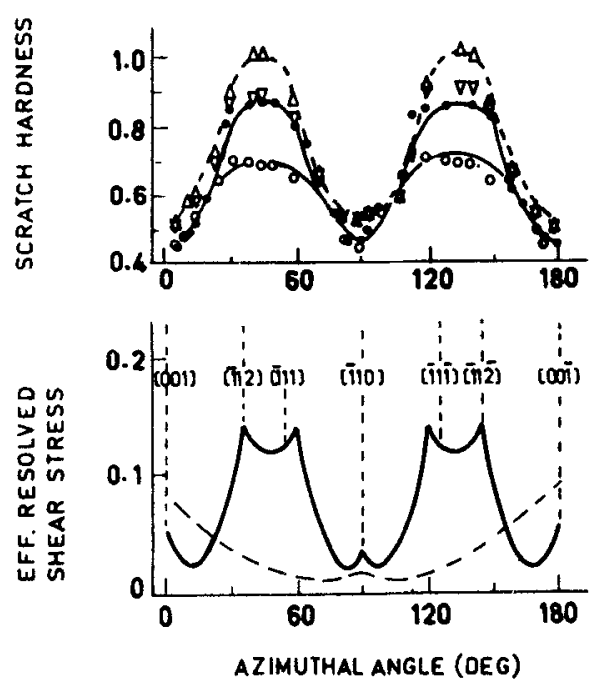

(b)

Figure 6. The measured scratch hardness results for copper. The indentor used was diamond with a $120^{\circ}$ cone and an edge-leading $136^{\circ}$ pyramid, and with a normal load of $500 \mathrm{~g}$. (a) (001) surface and (b) (110) surface. The bottom curves represent the calculated stress values along various directions, all lying in the plane on which measurements are made (Brookes 1981). 
above anisotropy can emerge from the calculation of the cohesive energy. The structure-dependent term $U_{\mathrm{BS}}$ in the total energy (equation (9)) must therefore be subjected to a closer examination. This involves analysing the band structure and Fermi surface in the presence of an applied stress. A beginning has been made in Watts (1979). It appears that by calculating the electron density from these Fermi surface results, one can discuss the influence of external stress on the elastic constants and, consequently the hardness. It is suggested that this line of approach might help in the endeavour to correlate the anisotropy in hardness with the anisotropy in the Fermi surface.

\section{Strengthening mechanisms: role of electron mediated processes}

There are a number of processes, well-known to metallurgists, available for enhancing the strength of elemental metals. The chief methods are (Peckner 1967): (a) dislocation strengthening, (b) strain hardening, (c) solid-solution hardening, (d) precipitation hardening, (e) dispersed-phase strengthening, (f) diffusionless (martensitic) transformation strengthening, $(\mathrm{g})$ superlattice strengthening, and $(\mathrm{h})$ radiation hardening. If we ask whether a definite electron-mediated effect has been identified with the strengthening mechanism in any of the above processes, the answer is a tentative 'yes' for mechanisms (c) and (g). We therefore start with the discussion of solid-solution strengthening.

\subsection{Solid-solution strengthening}

The simplest method of strengthening or hardening is by alloying the host element with other suitable elements/impurities. According to Conrad (1981), the solubility of the impurities will have a different value in the vicinity of dislocations than in other regions, and that the solute distribution will be such as to minimize the energy of the alloy. As the dislocations move under external stress this minimum energy configuration of the impurities is disturbed, raising the energy; the impurities thus tend to act as pinning centres for dislocations. The actual detailed mechanism could either be elastic or electronic in origin. (Ultimately, of course, elastic energy can be traced to an electronic origin). The problem of solution hardening in alloy physics can be related to that of calculating the change in energy of an impurity as its nearest neighbours vary their relative positions. The impurities are assumed to be arranged such that the distortion around the impurity is part of a macroscopic and uniform distortion of the surrounding host. The distortion is produced by first considering a single impurity in the host, undistorted except for the elastic distortion produced by a possible size mismatch. Thus a macroscopic and uniform distortion $\Delta S$ is applied to the host so as to produce the desired distortion around the impurity. Changes in the energy of the system as a function of the distortion are calculated and $\Delta E_{i}$, the part due to the presence of the impurity, is isolated. The interaction between the impurities is neglected (assuming the impurity to be sufficiently dilute). The magnitude of $\Delta E_{i} / \Delta S$ is a measure of the solution strengthening; the larger it is, the greater is the solution strengthening. Figure 7 shows the strengthening coefficient for a few Ti-based alloys (Collings and Gegel 1975). It can be deduced from these figures that the solid solution strengthening increases roughly in proportion to the distance between the columns in the periodic table of the solvent and solute. In $\mathrm{Ti}$, when such an impurity is added, its lack of $d$ character causes a larger perturbation in the wave function of the system in its vicinity. 


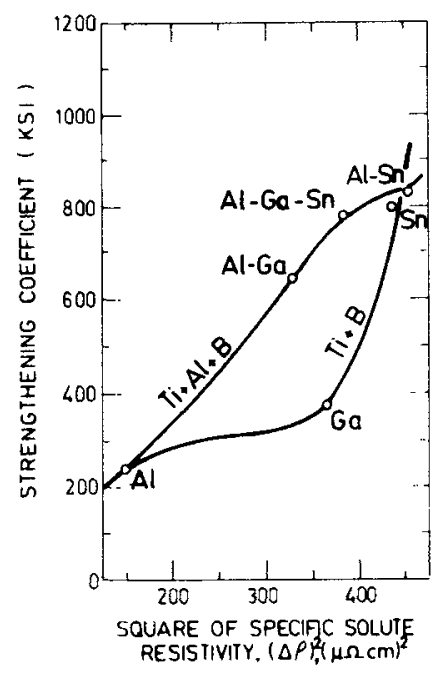

Figure 7. Correlation between the strengthening coefficient $(\mathrm{d} \sigma / \mathrm{d} C)$ (equivalently, $\left.\mathrm{d} E_{i} / \mathrm{d} a\right)$ and the electrical resistivity for a set of $\mathrm{Ti}+B$ alloys and a set of $\mathrm{Ti}+\mathrm{Al}+B$ alloys $(B=\mathrm{Ga}, \mathrm{Sn}$, etc.) (Collings and Gegel 1975).

Assuming that the wavefunction in the vicinity of an atom is the same as it is in the atomic configuration, the problem is simplified by imagining the perturbation introduced by the impurity to be spherically symmetric and highly localized. This then permits the definition of phase shifts introduced by the presence of the impurity. The phase shift (for a general $l$-character: $l=s, p, \ldots$ ) has the form

$$
\delta_{l}=\pi Z_{l} /(4 l+2)
$$

where $Z_{l}$ is the difference in the number of valence electrons between the impurity and the host. The introduction of the impurity and the corresponding phase shifts disturbs the wave function in the vicinity of the impurity. The distortion is appreciable and causes a given Bloch state to scatter into other states and decay with time. This leads to electrical resistivity; and indeed, a significant correlation has been found in experimental studies between the increase in electrical resistivity $\Delta \rho$ and the solution hardening. It is of interest to look at the form of the final expression for the strengthening coefficient $\mathrm{d} E_{i} / \mathrm{d} a($ Stern 1975)

$$
\frac{\mathrm{d} E_{i}}{\mathrm{~d} a}=\frac{1}{6 \pi} \frac{\mathrm{d} W}{\mathrm{~d} a}\left[E_{i}+\frac{2}{\pi} \int_{-\infty}^{0} \frac{\varepsilon_{0} I(\varepsilon) \mathrm{d} \varepsilon}{\left\{1-\varepsilon_{0} R(\varepsilon)\right\}^{2}+\left\{\varepsilon_{0} I(\varepsilon)\right\}^{2}}\right] .
$$

This is entirely equivalent to $\mathrm{d} \sigma / \mathrm{d} C$, the variation of the conductivity with the impurity concentration. In (13), $W$ is the band width and $a$ is the lattice parameter. ( $\varepsilon$ is the reduced energy parameter, $E / W$. $\varepsilon_{0}$ is six times the ground state energy of the impurity in units of $W . R$ and $I$ are certain functions that involve the phase shifts. As $\mathrm{d} W / \mathrm{d} a$ in (13) is independent of the impurity, the variation of interest is the expression in square brackets. If the impurity forms a bound state level below the bottom of the band $\left(U_{0} \ll-1\right)$, then $\left(\mathrm{d} E_{i} / \mathrm{d} a\right)$ is proportional to $U_{0}$; thus larger the magnitude of $U_{0}$, greater is the $\mathrm{d} E_{i} / \mathrm{d} a$, and greater the solid solution strengthening. 
Thus, it seems that an impurity with an electronic structure very different from that of the host causes a large perturbation of the wavefunction of the latter, leading to large phase shifts and consequently to high solution hardening. It would be worthwhile to measure the changes in the various elastic constants of the alloy with impurity concentration, in particular to confirm the expected increase in elastic constants with the addition of impurities of the above type (Conrad 1981).

\subsection{Superlattice strengthening}

In a solid solution, the atoms of the different components of the alloy form a common crystal lattice, generally that of the solvent. The metallic type of bond is found in all metal-based solid solutions regardless of the type of bond peculiar to the solute in the solid state. Nonmetallic atoms $(\mathrm{C}, \mathrm{N}, \mathrm{Si}$, etc.) do not change the metallic bond of metallic solid solutions. In a number of metallic systems $(\mathrm{Cu}+\mathrm{Au}, \mathrm{Fe}+\mathrm{Al}, \mathrm{Fe}+\mathrm{Si}, \mathrm{Ni}+\mathrm{Mn}$, etc.) the atoms of both components occupy entirely definite positions in the solid solution lattice if the alloy has been slowly cooled or has been heated for a long time at a definite temperature. Such ordered solid solutions are distinct from those in which the components assume statistically random positions in the lattice. This new order superimposed on the solvent lattice results in a structure called a 'superlattice'. Ordered solid solutions occur when the ratios of the alloyed components (in atomic \%) are equal to whole numbers, and are designated $\mathrm{Cu}_{3} \mathrm{Au}, \mathrm{Fe}_{3} \mathrm{Al}$ etc. The formation of an ordered solid solution is accompanied by a sharp fall in electrical resistivity and a sharp increase in hardness and strength.

The 'order-disorder' compounds are regarded as intermediate phase between solid solutions and chemical compounds. Much work has been done on these alloys (Warlimont 1974), including the correlation between their mechanical properties (in particular, the strength) and their electronic structure. Experimentally, correlations are found between a typical strength attribute, namely, hardness, and the so-called shortrange-order (SRO) parameter, which is related to the ordering energy $V(k)$. The SRO parameter can be determined from diffuse $x$-ray scattering measurements, as in these measurements one really probes the chemical nature of the nearest neighbours of an atom, and thus the sRO. Interesting Fermi surface effects giving rise to satellites in the diffuse x-ray scattering have been seen (Scattergood et al 1970), and thus the sRo and the Fermi surface effects in these compounds can be imagined to be related. If only the connection between any elastic constant (or any other suitable strength attribute) and the SRO parameter, can be established, the correlation between the strength and electronic structure in this mode of strengthening can be understood. This last step constitutes the lacuna in this area of investigation.

\section{Summary}

Elucidating the physical basis of one of the important mechanical attributes of a solid, namely, its strength, continues to be a challenging problem. Much success has, no doubt been achieved using the dislocation idea. We have examined the problem of understanding the strength of a crystalline solid on the basis of its electronic properties. The attempts that have been made so far adopted the view that cleavage can be regarded as the exact opposite of cohesion. This enables one to use in full the electronic theory of the cohesion of solids. The strength values calculated on this assumption agree by and 
large with the limited experimental values available. However, as pointed out in $\S 3$, there is an order of magnitude difference between the calculated and measured strains at failure. Also, it is true that the way the unit cell is assumed to deform in calculating the strength is not realistic. There is scope for refinements here, both in terms of allowing for more realistic deformations as well as extending the calculation to non-cubic symmetries of the unit cell. Nevertheless, it appears that as far as the problem of cleavage is concerned, well-known dislocation mechanisms and dislocation-crack-tip interactions are adequate to account for the strength of materials. The electronic theory (in the form of a phase shift analysis) meets with reasonable success when applied to the solution-strengthening problem. Systematic measurements of the elastic constants of sequences of alloys with, say, titanium as the host metal will be valuable. In the case of order-disorder compounds, an analysis of the relationship between the short-rangeorder parameter and the elastic constants (and thus the strength) would be very desirable. We have not commented in this article on the possible correlations that may exist between the electronic structure and the other strengthening mechanisms listed in $\S 6$.

\section{Acknowledgement}

The author thanks Dr G Venkataraman for suggesting this problem.

\section{References}

Beachem C D 1978 in Proceedings of the Second International Conference on Mechanical Behaviour of Materials (Ohio, Metals Park, ASM) 322ff

Brenner S S 1956 J. Appl. Phys. 271484

Brookes C A 1981 Philos. Mag. A43 529

Carlson A E, Gelatt C D and Ehrenreich H 1980 Philos. Mag. A41 241

Collings E W and Gegel H L 1975 Physics of solid solution strengthening (New York: Plenum Press)

Conrad H 1981 Prog. Mater. Sci. 262

Esposito E, Carlsson D D, Ehrenreich H and Gelatt C D (Jr) 1980 Philos. Mag. A41 251

Gerk A P and Tabor D 1978 Nature (London) 271732

Gilman J J 1969 Micromechanics of flow in solids (New York: McGraw Hill)

Gilman J J 1973 J. Appl. Phys. 44982

Hall E O 1970 Yield phenomena in metals and alloys (London: Macmillan and Co.)

Harrison W 1966 Pseudopotentials in the theory of metals (Massachussetts: Benjamin Inc.)

Havner K S 1979 J. Mech. Phys. Solids 27393

Kelly A 1973 Strong solids (Oxford: Clarendon Press)

Macmillan N H 1972 J. Mater. Sci. 7239

McQueen R G and Marsh S P 1962 J. Appl. Phys. 33654

Milstein F 1982 in Mechanics of solids (eds) H G Hopkins and M J Sewell (Oxford: Pergamon Press) 417ff O'Neill H 1967 Hardness measurement of metals and alloys (London: Chapman and Hall)

Peckner D 1967 The strengthening of metals (New York: Reinhold Publishing Co.)

Scattergood R O, Moss S C and Bever M B 1970 Acta Metall. 181087

Stern E A 1975 in Collings and Gegel (1975)

Warlimont H 1974 Order-disorder transformations in alloys (Berlin: Springer-Verlag)

Watts B K 1979 J. Phys. F9 1565

Westbrook J H and Conrad H 1973 The science of hardness testing and its research applications (Ohio, Metals Park, ASM)

Wilkes P 1973 Solid state theory in metallurgy (London: Cambridge University Press) 


\section{Discussion}

K R Rao: Could you comment on the nature of the electronic systems you are considering and their relation to the strength of materials?

K Govinda Rajan: The kind of electrons being considered will be specified once the potential is specified. It may also be useful to think in terms of electron charge densities around ion cores and their distortion during loading to understand strength phenomena.

A P Pathak: Instead of talking about the "effects of electronic structure on strength", it appears to be preferable to consider the correlation of electronic and mechanical properties. After all, all cohesion in solids is due to electronic structure (either orbital core electrons or conduction/valence electrons), and strength must necessarily be due entirely to electronic structure. Evidently the strength does not depend simply or directly upon, say, the band gap or the conduction electron density (as do the electrical properties), but on combinations of these properties, the core electrons, and of course the structure of the solid. Would you like to comment on this?

Govinda Rajan: In the exhaustive review by K A Gschneider (Solid State Physics, Vol. 16), one finds graphs of electronic properties (resistivity, valence electrons per atom, etc.) vs mechanical properties (yield strength hardness) for elemental metals. No significant microscopic picture, however, has emerged. What I have attempted instead is to identify-from the plethora of mechanical properties-the "strength" attribute, and to discuss the role of electronic structure in this context. This is because the strength parameter is, in a sense, the final stage of the mechanical response of a solid, and thus should be amenable to simple calculations. Even this view in which, for instance, cleavage is imagined to be the exact opposite of cohesion has met with serious objection from the metallurgists, as I have taken care to point out

G Venkataraman: It might be interesting to measure the hardness of a semiconductor after pumping electrons into the conduction band by, say, using a laser. This would also have practical relevance to devices like GaAs diode lasers. 